\title{
Imaging performance of a miniature integrated microendoscope
}

Jeremy D. Rogers

Sara Landau

Tomasz S. Tkaczyk

Michael R. Descour

College of Optical Sciences

University of Arizona

Tuscon, Arizona

E-mail: jdrogers@optics. arizona.edu

Mohammed S. Rahman

Rebecca Richards-Kortum

Department of Bioengineering

Rice University

Houston, Texas

Ari H. O. Kärkäinen*

Braggone Oy, Kaitovayla 1

FIN-90570 Oulu, Finland

Todd Christenson

HTmicro

Albuquerque, New Mexico

\begin{abstract}
An integrated miniature multi-modal microscope (4M device) for microendoscopy was built and tested. Imaging performance is evaluated and imaging results are presented for both fluorescence and reflectance samples. Images of biological samples show successful imaging of both thin layers of fixed cells prepared on a slide as well as thick samples of excised fixed porcine epithelial tissue, thus demonstrating the potential for in vivo use. () 2008 Society of Photo-Optical Instrumentation Engineers. [DOI: 10.1117/1.2978060]
\end{abstract}

Keywords: microscopy; endoscopy; micro-optics; optical testing; modulation transfer functions; medical imaging.

Paper 07181RR received Jun. 9, 2007; revised manuscript received Apr. 30, 2008; accepted for publication May 6, 2008; published online Sep. 15, 2008.

\section{Introduction}

The American Cancer Society reports that cancer is the second leading cause of death in the United States. ${ }^{1}$ The successful treatment of cancer often depends specifically on its early detection. Early detection of cancer and its precursors is possible using microscopic inspection of tissues, but this typically requires a biopsy of the tissue and preparation of the excised tissue for histological analysis. This procedure is often painful, expensive, and inconvenient, which limits its use.

If microscopic inspection can be performed in vivo without the need for a biopsy, the inspection of tissues at risk for cancer can be more easily facilitated. The development of a miniature microscope for microendoscopy is the goal of the research reported here. In this paper, we evaluate the imaging performance of an integrated miniature microscope by measuring the modulation transfer function (MTF) and present images of fixed biological samples acquired by the microendoscope to demonstrate its ability to image the cellular structure of tissue. The instrument is designed to image in fluorescence or reflectance, in widefield, or with optical sectioning using structured illumination. ${ }^{2}$ This multi-modal miniature microscope is referred to as the $4 \mathrm{M}$ device. A rendering of the complete 4M device conceptual design is shown in Fig. 1.

Optical sectioning using endomicroscopy has been the subject of research for several decades, and commercial instru-

Address all correspondence to J. D. Rogers, College of Optical Sciences, 1630 E. University Blvd., Tucson, AZ 85716. Tel: 847-467-0393; Fax: 520-626-3389. Email: jdrogers@optics. arizona.edu

*Also at University of Oulu ments are in use today. ${ }^{3-6}$ Indeed, many groups are actively pursuing various endomicroscopy techniques that show great potential for overcoming the many challenges of endomicroscopy. ${ }^{7-11}$ Our design includes only components that are fabricated using some form of lithography or printing and can be manufactured via mass production. Furthermore, the components are assembled using zero alignment to take advantage of the alignment features incorporated into the components. This method of assembly demonstrates the potential to eliminate the costly assembly step of optical system manufacture. The long-term intention is to make an instrument that would be very cheap to produce or even be disposable. Although not all components of the 4M device concept have yet been fully optimized and implemented, an important step in validating the technology is the evaluation of imaging performance of the unconventional optical and optomechanical design.

Our previously published works presented the concept, design, and fabrication technology used in the $4 \mathrm{M}$ device, including descriptions of the mounting method and conceptual system design, ${ }^{12-15}$ printed lens fabrication, ${ }^{16}$ optical design for stray light reduction, ${ }^{17}$ detector design, ${ }^{18}$ and a method of optical sectioning. ${ }^{19}$ The emphasis of this paper is the quantitative analysis of imaging performance and the demonstration of imaging cultured cells and prepared tissue in vitro.

1083-3668/2008/13(5)/054020/6/\$25.00 @ 2008 SPIE 


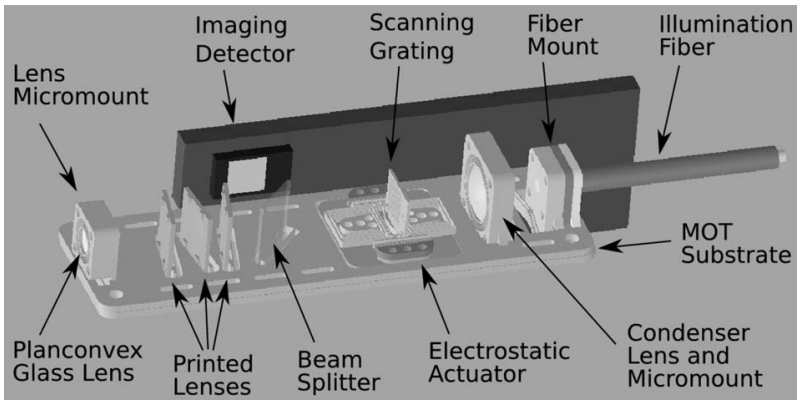

Fig. 1 Rendered design of the 4M device that illustrates optical components mounted on the MOT substrate. In the future completed device (not shown), the image sensor is to be mounted on a PCB and attached to the $4 \mathrm{M}$ device walls.

\section{Design Overview}

The design of the $4 \mathrm{M}$ device was restricted by several requirements that must be carefully balanced to achieve the imaging goals. To be useful as an endoscopic device, the instrument should be no more than a few millimeters in diameter. The magnification of the $4 \mathrm{M}$ device should be large enough to allow imaging of the cellular structure. To use the device in vivo, the objective must be capable of working in contact with body fluids (i.e., in immersion). To enable optical sectioning, the device must incorporate a scanning mechanism. For the device to work in reflectance mode (using epiillumination), the $4 \mathrm{M}$ device must be designed to limit and reduce the internal optical reflections to provide the contrast needed for cellular imaging. These requirements were incorporated into the design of the $4 \mathrm{M}$ device and resulted in the system properties described next.

The $4 \mathrm{M}$ device has a numerical aperture (NA) of 0.4 and a magnification of -4 that, when coupled with a custom CMOS imaging sensor design using $4 \times 4-\mu \mathrm{m}$ pixels, provides $1-\mu \mathrm{m}$ sampling of the object. ${ }^{18}$ The objective includes a $1-\mathrm{mm}$ plano-spherical glass lens mounted in a custom precision micromount and three aspheric microlenses printed via grayscale lithography in hybrid sol-gel glass. ${ }^{16}$ All optical elements are antireflection (AR) coated to reduce reflections and increase efficiency. The printed lenses utilize asymmetric tilted surfaces to reduce the adverse effect of stray light during reflectance imaging. ${ }^{17}$ The device design incorporates a scanning grating built using micro-electro-mechanical systems (MEMS) fabrication technology. ${ }^{20}$ The grating actuator is an electrostatic combdrive actuator designed to scan the grating in resonance. The illumination is provided by a highbrightness light-emitting diode (LED) coupled to a multimode fiber. The light from the fiber is collected by a $2-\mathrm{mm}$ planospherical glass condenser lens mounted in a precision micromount. A beamsplitter or dichroic is used to fold the image onto the CMOS detector. The components of the $4 \mathrm{M}$ device are all assembled onto a micro-optical table (MOT) ${ }^{12}$ fabricated with deep x-ray lithography (DXRL) and electroplating. The resulting device has a $250-\mu \mathrm{m}$-diameter field of view (FOV) and a working distance of $300 \mu \mathrm{m}$. The working distance allows for optical sectioning of epithelial cells below the tissue surface.

The device uses a $650-\mathrm{nm}$ peak wavelength for reflectance imaging and 450-nm excitation with 655-nm emission for fluorescence imaging. Although the optical system is not achromatic, the difference in imaging performance at each wavelength is primarily a change in focal length. Since the system does not simultaneously image multiple wavelengths, the effect of this change in focus can be accounted for by a simple change in the object plane within the tissue or by adjusting the position of the scanning grating within the device. There is no need for two completely different optical designs, and during construction the device can be assembled either for reflectance imaging using a beamsplitter or for fluorescence imaging using a dichroic and a different grating position. The source used for each mode is also substituted using a highbrightness LED of the appropriate peak wavelength, which is coupled to the system by a power delivery fiber.

\section{Fabrication and Construction}

The printed lenses were fabricated using a hybrid sol-gel glass developed specifically for this project. The material is described in detail in previous papers. ${ }^{21-24}$ The material was patterned to form lenses using a High Energy beam sensitive (HEBS) photomask available from Canyon Material, Inc. (San Diego, California). ${ }^{25}$ The mask optical density (OD) was encoded in 129 discrete steps that range from 0.125 to 1.562 ; a linear relationship between the OD and post-development material height was assumed. To pattern the lenses, the material was deposited onto 50-mm-diameter borosilicate glass substrates $^{26}$ made of \#1 coverslip glass approximately $150-\mu \mathrm{m}$ thick and then heated to $100^{\circ} \mathrm{C}$ on a hotplate to "wet" the substrate. The material was then spin-coated at $700 \mathrm{rpm}$ for 30 seconds and exposed through the HEBS mask using a Karl Suss MA-6 mask aligner for 15 seconds. The exposed element was developed in a 6:1 solvent solution of isopropanol and methyl isobutyl Ketone (MIBK) for 2 minutes. Upon removal from the developer bath, the part was rinsed for 5 seconds in ethanol and gently dried using a dry-nitrogen air gun. After development, the lens substrates were post-baked at $120^{\circ} \mathrm{C}$ for 2 hours. Finally, the lenses were AR coated by Accucoat (Rochester, New York) using a $\mathrm{V}$-coat centered at $650 \mathrm{~nm}$, then diced using a dicing saw into individual lens elements that measured $2 \times 2.5 \mathrm{~mm}$ and contained both lens surfaces and alignment features. ${ }^{16}$

The beamsplitter and dichroic mirrors used in the $4 \mathrm{M}$ device were made of additional \#1 coverslip glass substrates with appropriate coatings by Accucoat. The beamsplitter was AR coated on one side and coated with a $50 \%$ reflective coating on the other. The dichroic was made similarly. The beamsplitter or dichroic element was then scribed out of the substrate using a diamond scribe to produce a $2.5 \times 2.5 \mathrm{~mm}$ part that could be mounted in the MOT substrate $4 \mathrm{M}$ device. No alignment features were needed on these elements since the lateral position was not important and the position was constrained to the correct plane by the MOT substrate mounting slots. $^{27}$

The MOT substrate and MEMS components (including the grating actuator, fiber mount, and lens micromounts) were fabricated using a LIthographie, Galvanoformung, und Electroformung (LIGA)-like process by HTmicro of Albuquerque, New Mexico. The process used high-energy x-rays from a synchrotron radiation source to expose polymethylmethacrylate (PMMA) plastic through a special x-ray mask. The 


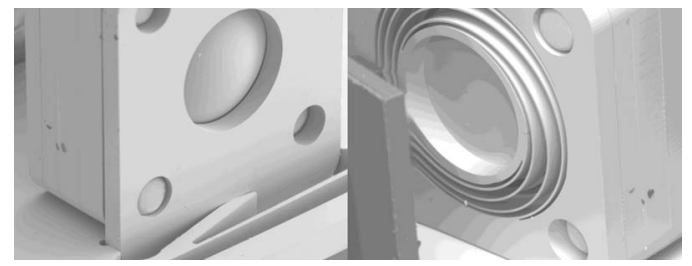

Fig. 2 SEM of the micromount lens holder assembled from metal LIGA parts. The lens aperture on the left is $0.8 \mathrm{~mm}$ and centers the spherical lens surface. A spring-loaded back plate shown at the right secures the lens. These layers and spacer layers in between are stacked and pinned together. The total width of each mount is $2.0 \mathrm{~mm}$.

exposed PMMA was then developed and used as a mold for electroplating. During electroplating, the metal filled the mold to produce precise prismatic structures in metal with submicron accuracy of the shape. The metal parts were then released and assembled to form 3-D precision components such as the MOT, grating actuator, gratings, and micromount lens holders shown in Fig. 2.

Assembly of the system was primarily performed manually using tweezers and a stereoscope. The printed lens elements included small ridges and blocks surrounding the lens surface that provided precision positioning constraints for the elements relative to the MOT substrate. Each lens element was inserted with tweezers behind the cantilever springs of the MOT slots, and the spring provided the force to snap the element into place. The micromounts had a simple tab with a precise width and depth to provide positioning constraints. The tab was inserted into the MOT slots, and a bistable spring was flipped to lock the tab in place. The micromounts were built from layers of LIGA parts and pinned together using precision pins and watchmaker's tools. Figure 3 shows the assembled $4 \mathrm{M}$ device positioned above a U.S. penny for scale.

\section{Testing and Performance}

An optical relay system was used to relay the image formed by the $4 \mathrm{M}$ device to a CCD camera. The custom CMOS sensor designed for the $4 \mathrm{M}$ device was still under development, so test data and preliminary images were taken using a relay system. The relay system consisted of two achromatic dou-

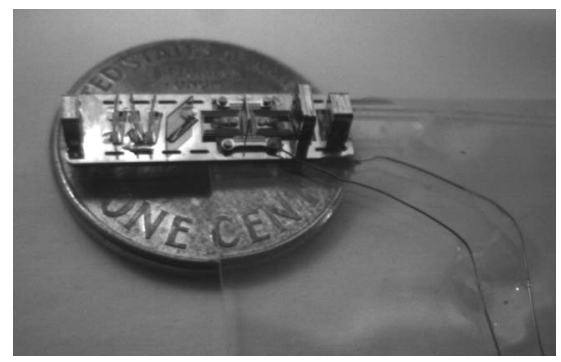

Fig. 3 Assembled $4 \mathrm{M}$ device shown above a United States penny for scale. The system incorporates the micromounted lens, hybrid printed lenses, beamsplitter, illumination optics, and fiber holder. The image is formed at the location where the detector will be integrated in future devices. This intermediate image is relayed by optical bench relay lenses to a CCD camera for testing purposes.

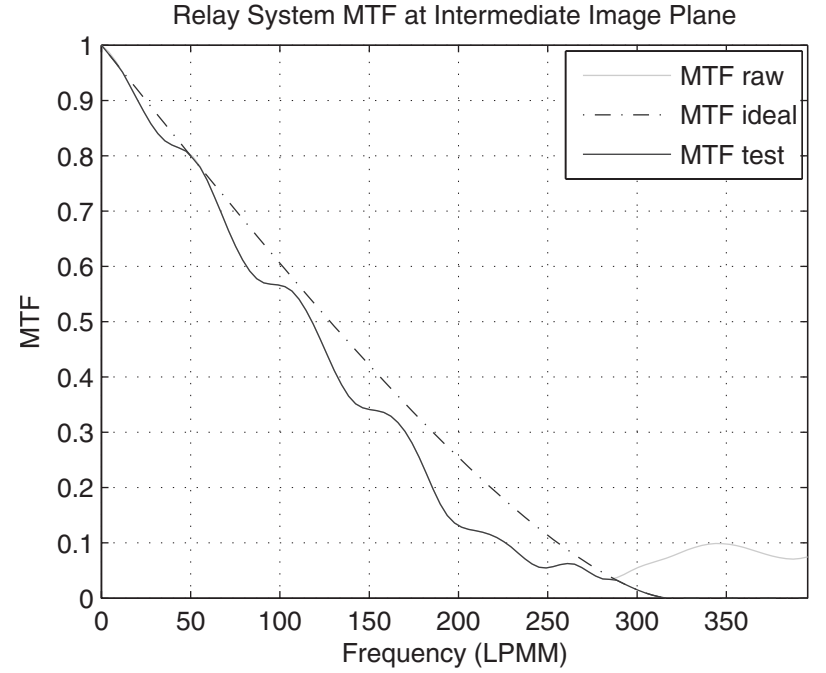

Fig. 4 Plot of the MTF of the relay optical system used in the $4 \mathrm{M}$ device imaging experiments. The dashed curve shows the ideal MTF for the relay system based on the NA. The solid dark line shows the calculated (measured) MTF of the relay system. The solid light line is the noise level of the measured raw data. Note that the frequency is in the object space of the relay system, which corresponds to the image space of the $4 \mathrm{M}$ device. Based on this measurement, the relay system was determined to be diffraction limited.

blets with focal lengths of $75 \mathrm{~mm}$ and $15 \mathrm{~mm}$. The lenses were arranged to relay the $4 \mathrm{M}$ image to the $\mathrm{CCD}$ with an additional magnification of -5 , giving a total system magnification of 20. An iris was positioned in the collimated space between the relay lenses at approximately the focal distance away from each lens to produce a double-telecentric relay system.

To quantify the imaging performance, the MTF of the $4 \mathrm{M}$ device was measured using a tilted-edge method. ${ }^{28}$ This method used an image of a slightly tilted edge (relative to the CCD pixel grid) to calculate the point-spread function and hence the MTF of the optical system. Since the image formed by the $4 \mathrm{M}$ device was relayed to a CCD camera using relay optics, the performance of the relay system alone was also measured. The resulting plot is shown in Fig. 4.

Once the relay optical system was shown to be diffraction limited, the $4 \mathrm{M}$ device was coupled to the relay system and the MTF measurement was performed on the combined 4M and relay optical systems. A typical MTF curve for one of the $4 \mathrm{M}$ devices (combined with relay) is shown in Fig. 5. From the measured MTF curve, a Strehl ratio of 0.67 was calculated for the device at $0.4 \mathrm{NA}$. The relay system contained an iris at the aperture stop location with a diameter of $2.3 \pm 0.2 \mathrm{~mm}$. The measured magnification of the combined system was 26.73, which, when combined with the uncertainty in the iris diameter, resulted in the NA range of 0.374 to 0.445 . The uncertainty in the NA led to an uncertainty in the calculated ideal MTF curve and resulted in a calculated Strehl ratio between 0.60 and 0.71 , depending on the NA.

\section{Imaging of Biological Specimens}

The long-term goal of our research is to utilize the $4 \mathrm{M}$ device to detect precancerous lesions in squamous epithelial tissues, 


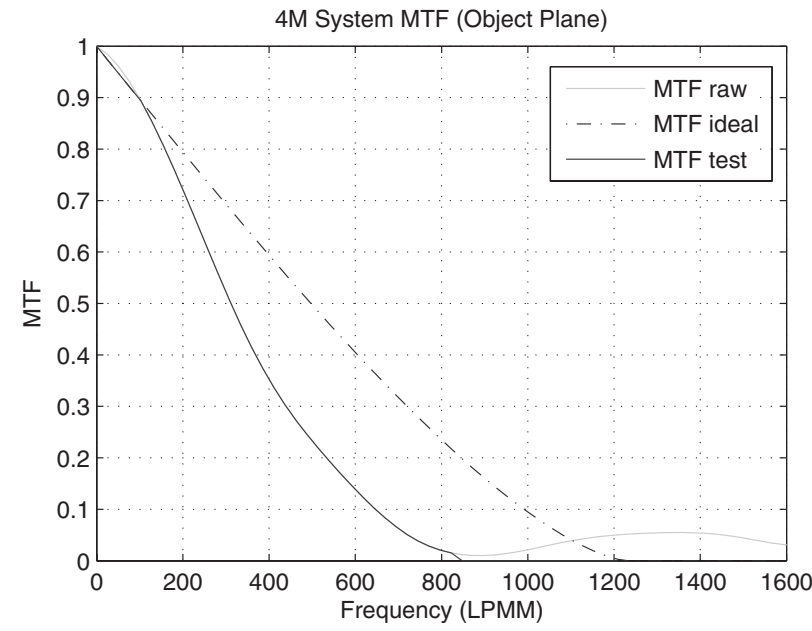

Fig. 5 Plot of the MTF of the combined relay and $4 M$ optical systems measured by the tilted-edge method. The dashed line represents the ideal MTF. The solid dark curve is the calculated MTF, and the solid light segment (raw data) was assumed to be noise and was blocked by a threshold level. The computed Strehl Ratio was 0.67 for the device with $0.4 \mathrm{NA}$.

especially in the oral cavity and cervix. To image the morphological and biochemical changes associated with precancer in these sites, multiple imaging modalities may be required. Thus, we considered two 4M devices with different modalities for imaging relevant biological specimens: one setup with a fluorescence imaging capability, and the other with a reflectance imaging capability. The differences between the two systems were the illumination sources and the beamsplitter coatings, as described earlier. To evaluate the performance of the $4 \mathrm{M}$ device in each of the modalities, we used exogenous contrast agents to label our biological specimens. The contrast agents provided two advantages in our study: an increased SNR of the samples compared to their native contrast, and molecular imaging of specific biomarkers of our interest, specifically the epidermal growth factor receptor (EGFR) in human oral precancerous cells.

For fluorescence imaging with the $4 \mathrm{M}$ device, we chose to label our samples with quantum dots. Quantum dots are highly stable semiconductor nanocrystals with a high quantum yield, which results in a much higher signal and SNR than typical autofluorescence signals from tissue. Moreover, their emission wavelengths are tunable with their size, allowing one to image in the near-infraRed (NIR) region of the spectrum where the penetration depth of light in tissue is higher. For this study, we chose CdSe quantum dots (QD655, Invitrogen), which have a broad excitation range from 300 to $500 \mathrm{~nm}$ and a narrow emission peak at $655 \mathrm{~nm}$. For reflectance imaging with the $4 \mathrm{M}$ device, we chose to label our specimens with 40 to $55-\mathrm{nm}$ gold nanoparticles. Recently, gold nanoparticles were shown to be potential contrast agents for in vitro molecular imaging of precancerous cells by Sokolov et al. ${ }^{29}$ and El-Sayed et al. ${ }^{30}$ The particles have the ability to resonantly scatter light in the visible and NIR wavelengths depending on their size and shape. We followed the protocol provided in Ref. 29 to synthesize our 40 to $55-\mathrm{nm}$ gold particles, which had a peak scattering coefficient wavelength between 630 and $680 \mathrm{~nm}$.
Since our goal in this paper was to demonstrate the highresolution imaging ability of the $4 \mathrm{M}$ device optical system in relevant biological samples, we chose biological specimens that closely approximated those organ sites with a squamous epithelium where the $4 \mathrm{M}$ system is intended to be used-for example, the oral cavity and cervix. Due to the structural and biomolecular similarity between the oral cavity and cervix, we considered only oral epithelial cells and tissue for our experiments. As mentioned earlier, contrast agents were used for molecular imaging of EGFR molecules, which are transmembrane glycoproteins that are over-expressed in both oral and cervical premalignant cells and tissue. ${ }^{31}$ Initial experiments were carried out on 3-D tissue phantoms made from 1483 human oral carcinoma cells. Phantoms were prepared by resuspending approximately 10 million cells in a volume of buffered collagen type I according to a procedure developed by Sokolov et al. ${ }^{29}$ Collagen-cell suspensions were plated into 6.5-mm-diameter transwells and allowed to grow for 24 hours to allow the cells to develop a 3-D network within the collagen matrix. Prior to imaging, the phantoms were labeled with appropriate contrast agents.

To label the phantoms with quantum dots, an indirect twostep labeling procedure was followed. Prior to primary labeling, the phantoms were incubated in a $1 \%$ bovine serum albumin (BSA) solution for 15 minutes to block any nonspecific labeling. The phantoms were then incubated in a $500-\mu \mathrm{L}$ solution of biotinylated anti-EGFR (clone 111.6, LabVision). After 1 hour of primary labeling at $24^{\circ} \mathrm{C}$, excess antibodies were removed from the phantoms by washing them in phosphate buffered saline (PBS) two times.

A $500-\mu \mathrm{L}$ solution of $10-\mathrm{nM}$ streptavidin-coated quantum dots was prepared in a $1 \times$ PBS for secondary labeling. After 30 minutes of secondary incubation at $24^{\circ} \mathrm{C}$, the phantoms were again washed in PBS to remove unbound quantum dots. Appropriate control samples, including nonspecific biotinylated anti-human goat IgG (BA-3000, Vector) and secondaryonly labeling, were also prepared. To label phantoms with gold nanoparticles, antibody-gold conjugates were first prepared according to the protocol presented in Ref. 29. The procedure involved preparing a mixture of colloidal gold particles and anti-EGFR monoclonal antibodies at $0.10 \mathrm{mg} / \mathrm{mL}$ (clone 29.1, Sigma) in a 20-mM HEPES buffer with a subsequent addition of polyethylene glycol (PEG) and centrifuging the mixture at $5000 \mathrm{rpm}$ for 2 hours to collect the bound conjugates. The phantoms were then incubated in a $500-\mu \mathrm{L}$ solution of the prepared conjugates for 1 hour at $24{ }^{\circ} \mathrm{C}$ in the presence of $10 \%$ dimethyl sulfoxide (DMSO) and washed twice in PBS to remove the unbound conjugates. DMSO was used as a permeation enhancer for the contrast agents. Control phantoms were similarly incubated with a nonspecific mouse IgG antibody-gold conjugated solution and washed in PBS. All phantoms were fixed in $10 \%$ formalin after labeling to preserve their natural structural integrity prior to imaging with the $4 \mathrm{M}$ devices.

The imaging setup for both of the $4 \mathrm{M}$ devices were conducted on an optical relay system with a color CCD camera (EC01380, Prosilica) as mentioned earlier. In fluorescence mode, the gain setting of the CCD was increased to optimize the signal-to-background ratio. The CCD was color-balanced prior to the imaging experiment. All imaging experiments 


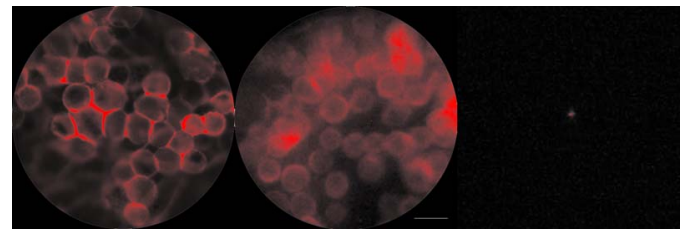

Fig. 6 Widefield images of cells labeled with fluorescent quantum dots imaged with the $4 \mathrm{M}$ device (center) and with a Zeiss Axiovert $100 \mathrm{M}$ for comparison (left). Cell membranes are clearly visible in each image. A control sample with nonspecific lgG labeling is shown on the right. The scale bar is $25 \mu \mathrm{m}$.

were carried out in a PBS immersion for index matching by bringing the $4 \mathrm{M}$ device lens into contact with the wet sample and then focusing back to the tissue plane of interest. The surface tension maintained the immersion of the $4 \mathrm{M}$ device to distances well beyond the working distance of the $4 \mathrm{M}$ device. The specimens were transferred to microscope slides and mounted on a micrometer for precise focusing of the sample on the $4 \mathrm{M}$ device. To compare the performance of the $4 \mathrm{M}$ devices, all samples were subsequently imaged with a Zeiss AxioImager Z1 microscope. A 20× dry objective lens with a NA of 0.6 was used for imaging, and images were acquired with a color CCD camera (AxioCam Mrc5, Zeiss). A quantum dot 655-nm filter set (Chroma Technology, U.S.) was used to image the fluorescence samples.

Figure 6 shows widefield fluorescence images of 1483 oral carcinoma cells in phantom acquired with the Zeiss AxioImager Z1 microscope and with the $4 \mathrm{M}$ device. The last image in the row is the $\mathrm{IgG}$ control specimen. The results show clear labeling of the targeted biomarkers around the cell membrane in our EGFR-positive sample, but no labeling in the control sample.

Figure 7 shows widefield reflectance images of the phantoms labeled with gold nanoparticles. The widefield images show the morphological pattern characteristic of single backscattering; however, the background associated with multiple light scattering from cell layers underneath reduces the image contrast. The 4M image is intermediate between the two-the single backscattering morphology is clearly visible in the center of the field, but the multiple scattering again reduces the

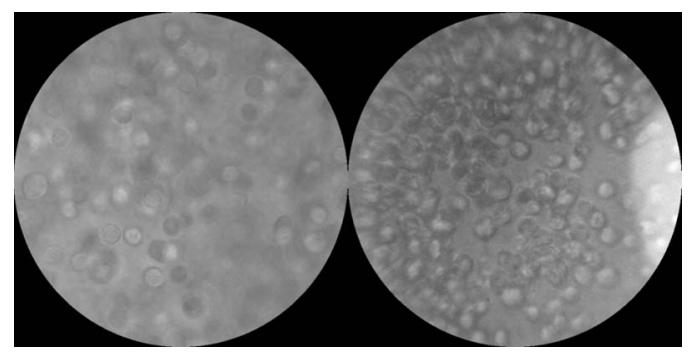

Fig. 7 Widefield image of cells imaged in reflectance labeled with gold nanoparticles. The image taken with the $4 \mathrm{M}$ device (right) shows the significance of the internal lens surface reflections. The bright region on the right of the image is due to a single surface reflection from an AR-coated lens. A similar image taken with a Zeiss Axiovert 100M is shown (left) for comparison. Black circular masks limit the FOV to $250 \mu \mathrm{m}$ for each image.

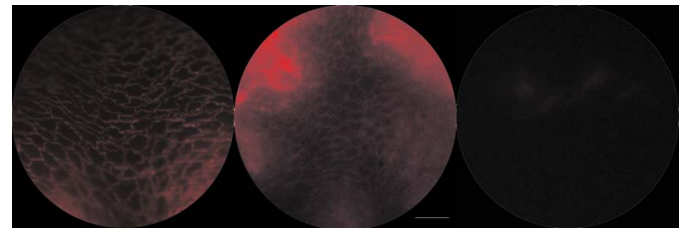

Fig. 8 Widefield fluorescent images of porcine epithelial tissue labeled with quantum dots taken with a Zeiss Axiolmager Z1 (left) and with the $4 M$ device (center). Squamous epithelial cells can be appreciated in both images. A control image is shown on the left. The scale bar is $25 \mu \mathrm{m}$.

image contrast somewhat. These results clearly demonstrate our ability to image backscattered light from cells in the current $4 \mathrm{M}$ device.

To demonstrate the imaging feasibility of the $4 \mathrm{M}$ device with more complex biological specimens, we chose porcine epithelial tissue because it is readily accessible and its morphological features are somewhat similar to those of human epithelial tissue. For sample preparation, fresh tissues were obtained from a local abattoir, and 6-mm punch biopsies were taken from the buccal mucosa of the porcine oral cavity. The tissues were then fixed in 10\% buffered formaldehyde for 24 hours to preserve their structural integrity for a longer period. The tissues were sliced transversely in 200 to $300-\mu \mathrm{m}$ thickness using a Krumdieck tissue slicer (Munford, Alabama). Tissue slices were subsequently labeled with contrast agents. For fluorescence imaging, the tissues were labeled with streptavin-coated $655-\mathrm{nm}$ quantum dots using the twostep indirect procedure described earlier. For positive labeling, biotinylated anti-swine IgG (BA-9020, Vector Lab) antibodies were used $(0.15 \mathrm{mg} / \mathrm{mL}$ in $1 \times$ PBS $)$. Samples were also prepared that included primary labeling with anti-mouse IgG (Labvision) and secondary-only labeling for control. We did not attempt to label the porcine tissues with gold nanoparticles due to the $4 \mathrm{M}$ device's lack of contrast in reflectance mode as experienced with the phantoms. Figure 8 shows widefield images of the porcine epithelial tissue taken with the Zeiss AxioImager Z1 microscope and with the $4 \mathrm{M}$ device. Although the signal was weak in the $4 \mathrm{M}$ image due to a limitation in the excitation power, squamous epithelium cells can still be appreciated from the image.

\section{Conclusions}

The results presented here demonstrate the ability to image fixed biological samples using our miniaturized microscope. Both reflectance and fluorescence imaging modalities can be used to acquire high-quality images of cells and epithelial tissues in vitro. Although furthur work is needed to use the device in vivo in a clinical environment, the results suggest that the $4 \mathrm{M}$ device can be used with targeted contrast agents to image the molecular features of cancer and its precursors. Furthermore, its application can potentially be extended to other areas of disease diagnosis where widefield microscopy is commonly used.

\section{References}

1. American Cancer Society "Cancer Facts and Figures, 2006," http:// cancer.org (2006). 
2. M. A. A. Neil, R. Juskaitis, and T. Wilson, "Method of obtaining optical sectioning by using structured light in a conventional microscope," Opt. Lett. 22, 1905-1907 (1997).

3. A. Gmitro and D. Aziz, "Confocal microscopy through a fiber-optic imaging bundle," Opt. Lett. 18, 565-567 (1993).

4. P. Delaney, M. Harris, and R. King, "Fibre-optic laser scanning confocal microscope suitable for fluorescence imaging," Appl. Opt. 33, 573-577 (1994).

5. R. Kiesslich, L. Gossner, M. Goetz, A. Dahlmann, M. Vieth, M. Stolte, A. Hoffman, M. Jung, B. Nafe, P. Galle, and M. Neurath, "In vivo histology of Barrett's esophagus and associated neoplasia by confocal laser endomicroscopy," Clin. Gastroenterol. Hepatol. 4, 979-987 (2006).

6. F. Jean, G. Bourg-Heckly, and B. Viellerobe, "Fibered confocal spectroscopy and multicolor imaging system for in vivo uorescence analysis," Opt. Express 15, 4008-4017 (2007).

7. T. Wang, M. Mandella, C. Contag, and G. Kino, "Dual-axes confocal microscopy with post-objective scanning and low-coherence heterodyne detection," Opt. Lett. 28, 414-416 (2003).

8. A. Rouse, A. Kano, J. Udovich, S. Kroto, and A. Gmitro, "Design and demonstration of a miniature catheter for confocal microendoscope," Appl. Opt. 43, 5763-5771 (2004).

9. H. Miyajima, K. Murakami, and M. Katashiro, "Mems optical scanners for microscopes," IEEE J. Quantum Electron. 10, 514-527 (2004).

10. M. Myaing, D. Macdonald, and X. Li, "Fiber-optic scanning twophoton fluorescence endoscope," Opt. Lett. 31, 1076-1078 (2006).

11. G. Tearney, M. Shishkov, and B. Bouma, "Spectrally encoded miniature endoscopy," Opt. Lett. 27, 412-414 (2002).

12. M. R. Descour, A. H. O. Karkkainen, J. D. Rogers, C. Liang, R. S. Weinstein, J. T. Rantala, B. Kilic, E. Madenci, R. R. RichardsKortum, E. V. Anslyn, R. D. Dupuis, R. J. Schul, C. G. Willison, and C. P. Tigges, "Toward the development of miniaturized imaging systems for detection of pre-cancer," IEEE J. Quantum Electron. 38 122-130 (2002).

13. J. D. Rogers, M. R. Descour, A. H. O. Kärkkäinen, R. J. Shul, C. G Willison, and C. P. Tigges, "Fabrication and assembly of miniature imaging systems using lithographically patterned microoptics and silicon microstructures," in OSA Topical Meeting on Diffractive Optics and Micro Optics (DOMO 2002), DTuC4 (2002).

14. J. Lee, J. D. Rogers, D. L. Nida, J. S. Aaron, K. V. Sokolov, R. R. Richards-Kortum, and M. R. Descour, "Multimodal miniature microscope (4M Device): novel methodology for multimodality tissue imaging in vivo," Proc. SPIE 4962, 168-176 (2003).

15. T. S. Tkaczyk, J. D. Rogers, M. Rahman, T. C. Christenson, S. Gaalema, E. L. Dereniak, R. Richards-Kortum, and M. R. Descour, "Multi-modal miniature microscope: 4M Device for bio-imaging applications-an overview of the system," Proc. SPIE 5959, 138$146(2005)$

16. J. D. Rogers, A. H. O. Kärkkäinen, T. Tkaczyk, J. T. Rantala, and M. Descour, "Realization of refractive microoptics through grayscale lithographic patterning of photosensitive hybrid glass," Opt. Express 12, 1294-1303 (2004).

17. J. D. Rogers, M. R. Tkaczyk, T. S. Descour, A. H. O. Kärkkäinen, and R. Richards-Kortum, "Removal of ghost images by using tilted element optical systems with polynomial surfaces for aberration compensation," Opt. Lett. 31, 504-506 (2006).

18. T. S. Tkaczyk, E. L. Dereniak, S. Gaalema, W. Bahn, S. Sun, T. Erickson, J. D. Rogers, T. C. Christenson, R. Richards-Kortum, and M. R. Descour, "High speed CMOS for structured illumination technique," Proc. SPIE 5726, 77-84 (2005).

19. T. S. Tkaczyk, M. Rahman, V. Mack, K. Sokolov, J. D. Rogers, R. Richards-Kortum, and M. R. Descour, "High resolution, molecular-specific, reflectance imaging in optically dense tissue phantoms with structured-illumination," Opt. Express 12, 3745-3758 (2004).

20. M. G. el Hak, ed., The MEMS Handbook, CRC Press, Boca Raton, Florida (2002).

21. J. T. Rantala, A. H. Kaerkkaeinen, J. A. Hiltunen, T. Kololuoma, and M. R. Descour, "New method to fabricate 3D optical structures into glass-ceramic films," Proc. SPIE 4284, 74-79 (2001).

22. J. T. Rantala, A. Karkkainen, J. A. Hiltunen, M. Keranen, T. Kololuoma, and M. R. Descour, "UV light induced surface expansion phenomenon of hybrid glass thin films," Opt. Express 8, 682687, June 2001.

23. A. H. Karkkainen, J. D. Rogers, G. E. Jabbour, J. T. Rantala, and M. R. Descour, "Recent progress in hybrid glass materials for microoptical component fabrication," Proc. SPIE 4455, 15-22 (2001).

24. A. H. O. Karkkainen, J. M. Tamkin, J. D. Rogers, D. R. Neal, O. E. Hormi, G. E. Jabbour, J. T. Rantala, and M. R. Descour, "Direct photolithographic deforming of organomodified siloxane films for micro-optics fabrication," Appl. Opt. 41, 3988-3998 (2002).

25. Canyon Materials, Inc., http://canyonmaterials.com.

26. ProSciTech, http://www.proscitech.com.au.

27. J. D. Rogers, Miniature Microscope Design and Construction Based on Tilted Rotationally Asymmetric Printed Lenses. PhD thesis, University of Arizona (2006).

28. A. P. Tzannes and J. M. Mooney, "Measurement of the modulation transfer function of infrared cameras," Opt. Eng. 34, 1808-1817 (1995).

29. K. Sokolov, M. Follen, J. Aaron, I. Pavlova, A. Malpica, R. Lotan, and R. Richards-Kortum, "Real time vital imaging of pre-cancer using anti-egfr antibodies conjugated to gold nanoparticles," Cancer Res. 63 (2003).

30. H. El-Sayed, X. Huang, and M. El-Sayed, Nano Lett. 5, 829-834 (2005).

31. E. Hsu, E. Anslyn, S. Dharmawardhane, R. Alizadeh-Naderi, J. Aaron, K. Sokolov, A. El-Naggar, A. Gillenwater, and R. Richards-Kortum, "A far red fluorescent contrast agent to image epidermal growth factor receptor expression," Photochem. Photobiol. 79(3), 272-279 (2004). 